\title{
PERFORMANCE OF MULTIPLE ERROR CORRECTION (MEC) SCHEME BASED HYBRID ARQ (HARQ) ALGORITHM FOR MAXIMIZING LIFETIME OF WIRELESS SENSOR NETWORK (WSN) FOR NATURAL DISASTER MONITORING
}

\author{
S. Razali, K. Mamat and S. K. Bashah ${ }^{*}$ \\ Faculty of Computer and Mathematical Sciences, UniversitiTeknologiMARA,40450 Shah \\ Alam, Selangor, Malaysia
}

Published online: 17 October 2017

\begin{abstract}
Natural disaster monitoring has become the critical area in Wireless Sensor Network to monitor the danger forthe accurate early warning to the public. The main issues such as energy-constrained and high error rates had become the utmost debates among the researchers. Hybrid ARQ was known as one of the error control protocols that outperform the existing ARQ and FEC. The existing HARQ might not be able to encounter the issues of network lifetime due to the advancement of this Sensor technology. Thus, we focused on the factors of Bit Error Rates and interference that riseto design the extension of HARQ that could lower the usage of energy and help toprolong the lifetime of the network. This paper proposed Multiple Error Correction algorithm that was based on the existing process of HARQ and presented the outcomes of implementing MEC towards the network of WSN.
\end{abstract}

Keywords: HARQ, wireless sensor network, lifetime, CDMA.

Author Correspondence, e-mail: samirahnasr@gmail.com

doi: http://dx.doi.org/10.4314/jfas.v9i5s.24 


\section{INTRODUCTION}

Wireless Sensor Network technology had contributed mainly in the field of monitoring such as in habitat, environment [1], agricultural, military, health [2], tracking and even in marine [3]. The sensor will detect any changes in the environments such as changes in temperature, humidity and even water levels and send the data from one node to another until reaching the base station (BS) or sink. The common wireless sensor network deployed wireless adhoc communication. However, there are many different approaches to implement WSN in terms of routing protocols, node deployment and even type of channel access. As for this paper, we apply WSN with Coded Division Multiple Access (CDMA) [4-6].

Previous research stated that CDMA has the advantages to support the applications withstrict latency requirements applications and high bandwidth. The CDMA technology was preferred to be used in WSN as it had the adequacy to reuse the frequency in which allows the network to be easily expanded or on the other hand, it supports high scalability of the network. Despite its advantages, CDMA can create some contentionif being applied in the congested and unconducive environment such as in the network with high number of nodes and high number of collisions. There was alsoa concernon Multiple Access Interference (MAI) that can lead to collision and further causeshigh number of bit errors. Thus, in order to overcome the problem of high Bit Error Rates (BER) in the network, the modification of error control protocol was the best solution for this matter.

ARQ and FEC have been known to aid the network with retransmission and error correction respectively. However, as these two error control protocols were used alone, there might foster unnecessary overheads. ARQ uses retransmission strategy in which the transmitter will retransmit the corrupted packet until the successful delivery were achieved. If there are high number of errors presented during transmissions, the network might be flooded with retransmission packets. This will increase the latency and lengthen the delaythen eventually will use more energy. FEC might not flood the network with retransmission packets or cause irrelevant congestion, however, the usage of FEC creates more complexity in terms of the decoding process and in some cases can also consume more energy [7].Hybrid Automatic Repeat Request (HARQ) were being studied and implemented in order to minimize the overheads of ARQ and FEC. HARQ is the conventional combination of ARQ and FEC. In 
HARQ, usually the Cyclic Redundancy Check (CRC) was usedfor error detection and error correcting codes (ECC) such as BCH and Reed-Solomon (RS) code was used to correct errors. When the retransmission is sufficient to correct corrupted packet, then the retransmission will be established by the sender instead of error correction using error correcting codes. This existing HARQ however does not tolerate with interference very well and the inaccurate usage of high error correcting capability in an unfavourable environment of CDMA WSN can cause high latency and might cause corruption in the data transfer. This high latency and BER can lead to the increasedin energy usage and degraded the lifetime of the sensors.

This paper proposed Multiple Error Correction (MEC) as an extension to the HARQ process in reducing BER and interference problem to lessen the energy consumption and indirectly, maximize the lifetime of the network. This paper also investigates the effect of MEC algorithm towards the performance of BER, energy consumption and latency in CDMA WSN. The rest of this paper is organized as follows.The MEC algorithm design was illustrated in section 2. In section 3, the WSN architecture and node deployment that imitated the node deployment in natural disaster monitoring were explained. The results were analyzed and presented in section 4 and were also discussed in details. Lastly, conclusion was stated in section 5 .

\section{MEC ALGORITHM DESIGN}

We had proposed Multiple Error Correction (MEC) algorithm that extend the existing process of HARQ. MEC divided the error correction schemes to correspond to the different network condition. The network condition was measured with Signal-to-noise ratio (SNR). We assumed as the SNR grows higher, the better the network condition. MEC divide the error correction schemes to correlate to various network conditions in order to minimize the use of unnecessary high error correcting capability of error correcting codes. This is because higher error correcting codes append more redundancy that adds more latency during the decoding processwhich uses more energy. The good network condition or uncongested network might not need powerful error correcting codes if only retransmissions were necessary. Fig 1 shows the block diagram of the process of MEC from sender to receiver. 


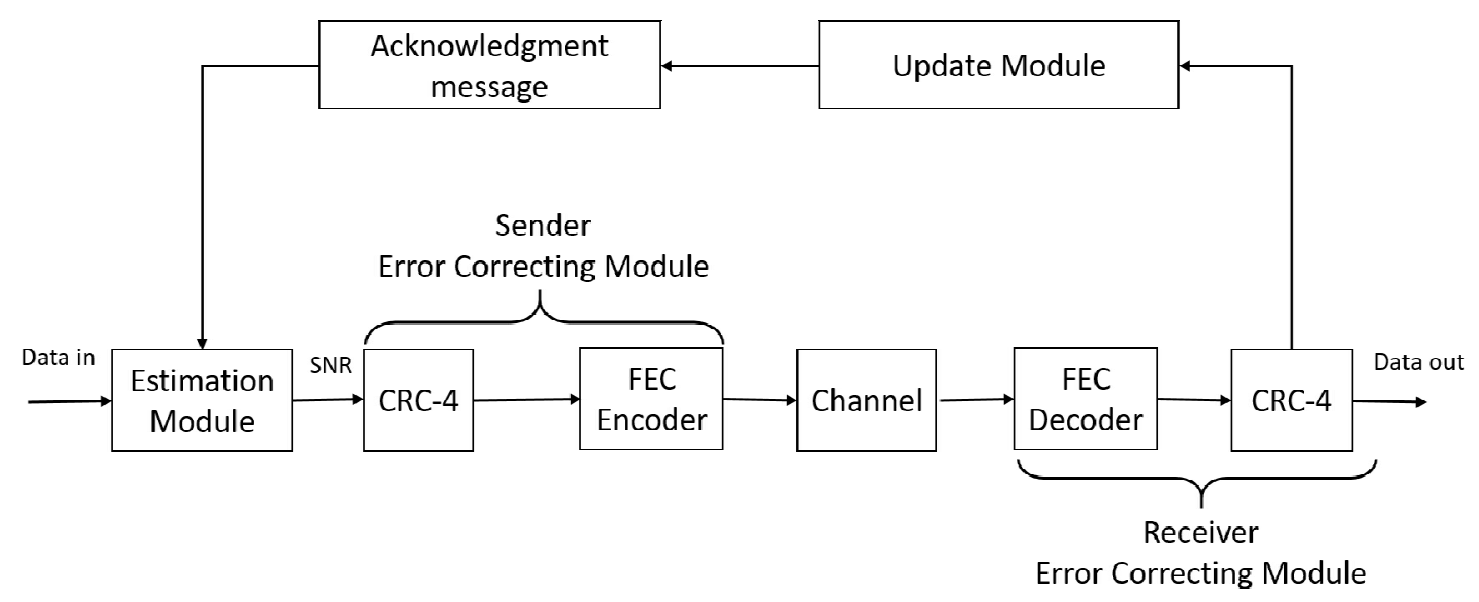

Fig.1. The block diagram of MEC algorithm the process from sender to receiver MEC algorithm consists of three modules such as the estimation module, the error correcting module and the update module as shown in Fig. 1. During the estimation module, the value SNR will be estimatedbefore the actual transmission was taking place. This SNR value will attune to the error correction scheme that will occur at the sender. For this paper, we had fixedthe range of SNR to be in between 1 to 20. In a real-time environment, the SNR value will change according to the environmental factors such as the configuration, routing protocols, shadowing, fading and the hardware that were used.

The error correcting module will startafter the SNR value had been determined. During error correcting module, the algorithm will decide on the error correction mode whether to only proceed with the retransmission or apply the ECC. MEC uses CRC-4 for error detection and $\mathrm{BCH}$ and RS for error correction. The transmit power also will be decided at the sender or receiver to optimize the effect of interferences. This transmit power will be increased or decreased depending on the network condition or congestion that might occur. The details of the error correction mode in the error correcting module are summarized in Table 1. 
Table 1. Error correction mode of error correcting module in MEC

\begin{tabular}{|c|c|c|}
\hline $\begin{array}{c}\text { Error } \\
\text { Correction Mode }\end{array}$ & SNR & Properties \\
\hline Lowest & For $17<\mathrm{SNR}<20$ & $\begin{array}{l}\text { Sender node, } \mathrm{S}_{\mathrm{i}} \text { prepare for retransmission with } \\
\text { Chase-combining }(\mathrm{CC}) \\
\text { Lower Pt by } 10 \%\end{array}$ \\
\hline Low & For $13 \leq \mathrm{SNR} \leq 16$ & $\begin{array}{l}\text { Encode data with } \mathrm{RS} \text { code with error correcting } \\
\text { capability, } \mathrm{t} \text { where } 1<\mathrm{t}<3 \\
\mathrm{Pt} \text { is maintained }\end{array}$ \\
\hline Medium & For $9 \leq \mathrm{SNR} \leq 12$ & $\begin{array}{l}\text { Encode data with } \mathrm{RS} \text { code with error correcting } \\
\text { capability, } \mathrm{t} \text { where } 4<\mathrm{t}<7 \\
\mathrm{Pt} \text { is maintained }\end{array}$ \\
\hline High & For $5 \leq \mathrm{SNR} \leq 8$ & $\begin{array}{l}\text { Encode data with } \mathrm{BCH} \text { code with error correcting } \\
\text { capability, } \mathrm{t} \text { where } 1<\mathrm{t}<3 \\
\text { Pt will be increased by } 5 \%\end{array}$ \\
\hline Highest & For $1 \leq \mathrm{SNR} \leq 4$ & $\begin{array}{l}\text { Encode data with } \mathrm{BCH} \text { code with error correcting } \\
\text { capability, } \mathrm{t} \text { where } 4<\mathrm{t}<7 \\
\text { Pt will be maintained by } 10 \%\end{array}$ \\
\hline
\end{tabular}

The update module aided the receiver to estimate and update the value of SNR before sending the acknowledgement (ACK) or negative acknowledgement (NACK) message back to the sender. The estimated value will prepare the sender to retransmit the message back to the receiver with different error correction schemes that correspond to the network condition at that time if the retransmission or correction needed.

\section{SYSTEM MODELS AND SIMULATION}

\subsection{Wireless Sensor Network Topology}

The wireless sensor network node deployment separated into two type of topologies such as uniform node deployment and non-uniform node deployment. The uniform node deployment follows the work of [5]for the purpose of evaluation. For uniform node deployment, the distances between nodes in the topology are the same. Fig. 2 shows the uniform node 
deployment in which $\mathrm{d} 1, \mathrm{~d} 2, \mathrm{~d} 3, \mathrm{~d} 4$ and $\mathrm{d} 5$ indicate the distance between the sender and receiver. For example, the distance between $\mathrm{d} 2$ to $\mathrm{d} 3$ such that node 28 to node 11 are the same.

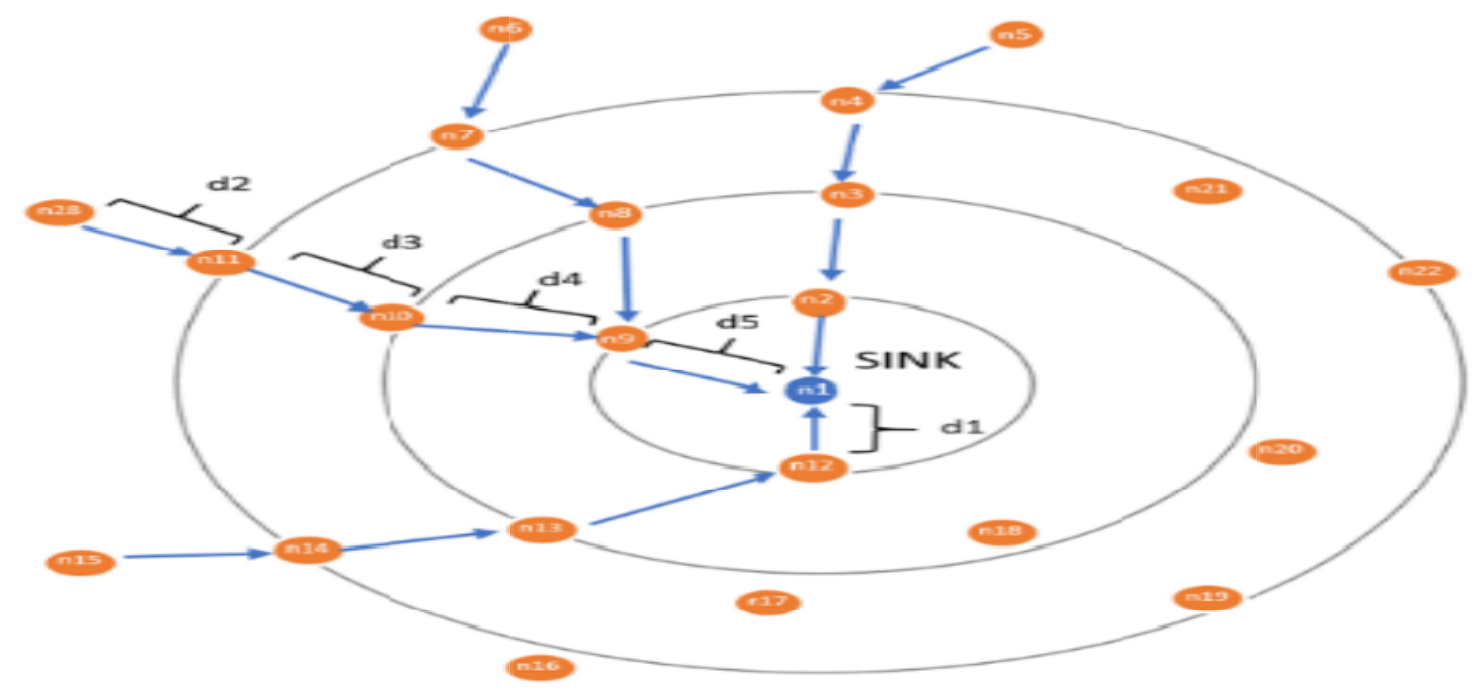

Fig.2. The topology of uniform node deployment [5]

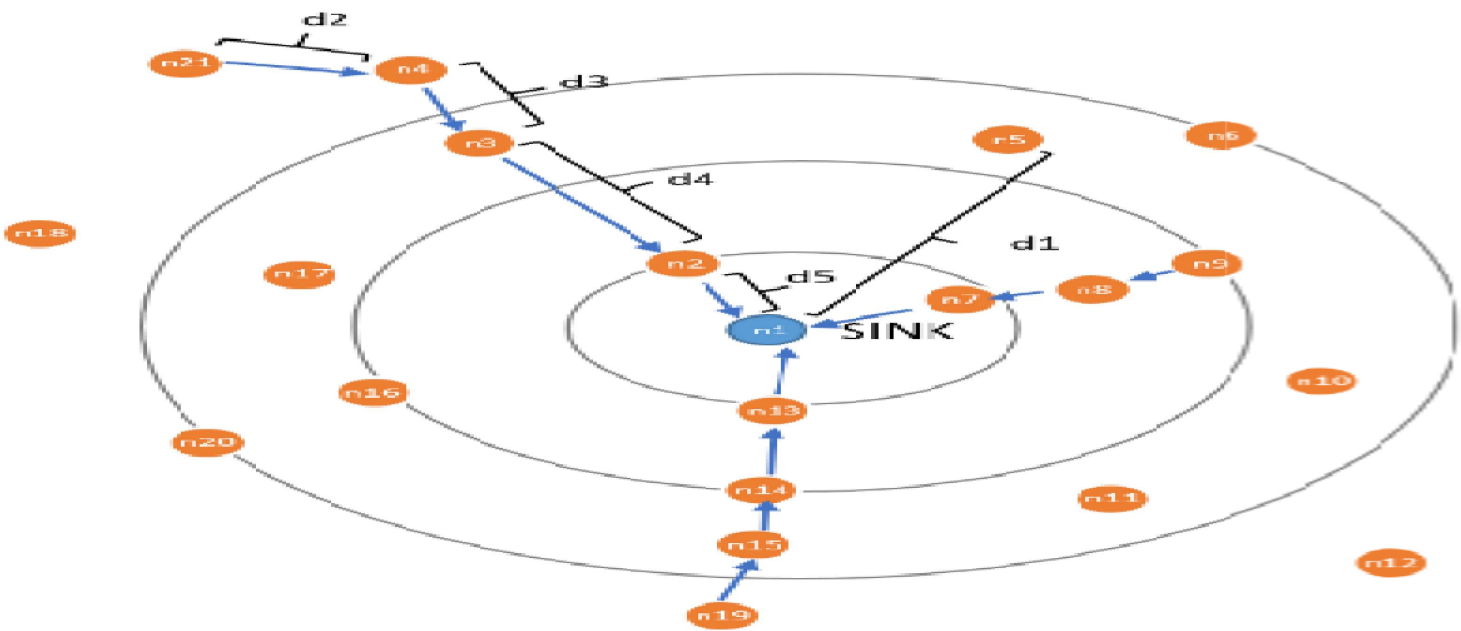

Fig.3. The topology of non-uniform node deployment

Fig. 3 shows the topology of non-uniform node deployment. We proposed the non-uniform node deployment to imitate the natural disaster monitoring such as volcanic eruption or flood detection system where the node will not necessarily be uniform in distance between each other as the geographical constraints and nodes are most likely to be placed at the different height levels of hills or riverside. For this topology, the distance between sender and receiver such that $\mathrm{d} 1, \mathrm{~d} 2, \mathrm{~d} 3, \mathrm{~d} 4$ and $\mathrm{d} 5$ are not the same. For example, the distance between node 3 and node 2 is $6 \mathrm{~m}$ while the distance between node 3 to node 4 is $3 \mathrm{~m}$. We had set that the 
minimum distance between nodes is $5 \mathrm{~m}$ while the maximum distance between the outer layer nodes to the sink is $20 \mathrm{~m}$.

\subsection{System Models}

The scenarios that were used throughout the experiments were divided into two groups such as in Table 2 and Table 3. Table 2 explained Scenario group A which consists of six scenarios with the same allocation of nodes with different measured distance and MEC error correction schemes. Scenario 1, scenario 2 and scenario 3 consists of uniform node deployment for the shorter distance such that the distance between sender to receiver or sender to sink, $\mathrm{d}$ is 5 meters. The error correcting schemes use the same error correcting capability, $\mathrm{t}$ of $\mathrm{BCH}$ codes and RS code where the codeword length, $\mathrm{n}$ is 63 was fixed and the data symbol, $\mathrm{k}$ were changed according to the error correcting capability, $\mathrm{t}$ in as shown in Table 2 throughout the experiments. Scenario 4, scenario 5 and scenario 6 consists of longer distance for uniform node deployment were $\mathrm{d}=20$ meter. The error correction schemes follow scenario 1 , scenario 2 and scenario 3 respectively.

Table 2. Scenarios A defined for simulation

\begin{tabular}{|c|c|c|c|}
\hline Scenarios & Description & $\begin{array}{c}\text { Error Correcting } \\
\text { Capability, } \mathbf{t}\end{array}$ & $\begin{array}{l}\text { Error Correction Schemes } \\
\text { for Error Correction Mode }\end{array}$ \\
\hline 1 & & $\mathrm{t}=3$ & $\begin{array}{c}\mathrm{BCH}(63,45) \\
\operatorname{RS}(63,57)\end{array}$ \\
\hline 2 & $\begin{array}{l}\text { Node deployment shorter } \\
\text { distance, } \mathrm{d}=5 \mathrm{~m}\end{array}$ & $\mathrm{t}=5$ & $\begin{array}{c}\mathrm{BCH}(63,36) \\
\operatorname{RS}(63,53)\end{array}$ \\
\hline 3 & & $\mathrm{t}=7$ & $\begin{array}{c}\mathrm{BCH}(63,24) \\
\operatorname{RS}(63,49)\end{array}$ \\
\hline 4 & & $\mathrm{t}=3$ & $\begin{array}{c}\mathrm{BCH}(63,45) \\
\operatorname{RS}(63,57)\end{array}$ \\
\hline 5 & $\begin{array}{l}\text { Node deployment with } \\
\text { longer distance, } d=20 \mathrm{~m}\end{array}$ & $t=5$ & $\begin{array}{c}\mathrm{BCH}(63,36) \\
\operatorname{RS}(63,53)\end{array}$ \\
\hline 6 & & $\mathrm{t}=7$ & $\begin{array}{c}\mathrm{BCH}(63,24) \\
\operatorname{RS}(63,49)\end{array}$ \\
\hline
\end{tabular}

While, Table 3 shows another of six scenarios for scenario group B with the same allocation 
of nodes in Table 2 but differ in the arrangement of error correction schemes use for MEC. The different allocation of error correcting capabilities in MEC is to study the effect of lower error correcting capability towards BER in the network. This is because, lower $t$ can optimize the latency and energy consumption but might have drawback towards the BER. However, applying the error schemes such in Table 3 might optimize BER as well as energy and latency all together.

Table 3. Scenarios B defined for simulation

\begin{tabular}{|c|c|c|c|}
\hline Scenarios & Description & $\begin{array}{c}\text { Error Correcting } \\
\text { Capability, } \mathbf{t}\end{array}$ & $\begin{array}{l}\text { Error Correction Schemes } \\
\text { for Error Correction Mode }\end{array}$ \\
\hline \multirow{2}{*}{7} & & $\mathrm{BCH} \mathrm{t}=3$ & $\mathrm{BCH}(63,45)$ \\
\hline & & $\mathrm{RS} \mathrm{t}=4$ & $\operatorname{RS}(63,55)$ \\
\hline \multirow{2}{*}{8} & Node deployment shorter & $\mathrm{BCH} \mathrm{t}=5$ & $\mathrm{BCH}(63,36)$ \\
\hline & distance, $\mathrm{d}=5 \mathrm{~m}$ & $\mathrm{RS} \mathrm{t}=6$ & $\operatorname{RS}(63,51)$ \\
\hline \multirow{2}{*}{9} & & $\mathrm{BCH} \mathrm{t}=7$ & ВCH $(63,24)$ \\
\hline & & $\mathrm{RS} \mathrm{t}=8$ & $\operatorname{RS}(63,47)$ \\
\hline \multirow{2}{*}{10} & & $\mathrm{BCH} t=3$ & $\mathrm{BCH}(63,45)$ \\
\hline & & $\mathrm{RS} \mathrm{t}=4$ & $\operatorname{RS}(63,55)$ \\
\hline \multirow{2}{*}{11} & Node deployment with & $\mathrm{BCH} \mathrm{t}=5$ & $\mathrm{BCH}(63,36)$ \\
\hline & longer distance, $\mathrm{d}=20 \mathrm{~m}$ & $\mathrm{RS} \mathrm{t}=6$ & $\operatorname{RS}(63,51)$ \\
\hline \multirow{2}{*}{12} & & $\mathrm{BCH} \mathrm{t}=7$ & $\mathrm{BCH}(63,24)$ \\
\hline & & $\mathrm{RS} \mathrm{t}=8$ & $\operatorname{RS}(63,47)$ \\
\hline
\end{tabular}

We simulate the topologies in Fig. 2 and Fig 3 in the environment of MATLAB r2016b. The details of variables that had been used for the configuration and simulation was shown in Table 4. We incorporated the use of Rayleigh Fading and Multi-Carrier CDMA. The binary data generated will be appended with CRC-4 bit for error detection. If the network condition determined the use of error correcting codes were crucial, then the encoded data will then be encoded once again with defined ECC in Table 2 and 3. Then, the BPSK modulation took place as well as spreading. The encoded data will be passed to the channel with Additive white Gaussian noise (AWGN) to the receiver. 
Table 4. Variables defined for simulation

\begin{tabular}{ll}
\hline \multicolumn{1}{c}{ Variables } & \multicolumn{1}{c}{ Details } \\
\hline Architecture & Multi-Carrier CDMA \\
Channel Model & Rayleigh Fading \\
Modulation & BPSK \\
Noise & AWGN \\
Error Detection & Cyclic Redundancy Check \\
& $($ CRC-4) \\
Error Correction Codes & Reed-Solomon (RS), \\
& BCH \\
Number of Nodes & $4,16,32,80$ \\
Bit Length (Bits) & $10000,20000,30000$ \\
Minimum Distance between Sender and Receiver (meters) & 5 meters \\
Maximum Distance between Sender and Receiver (meters) & 20 meters \\
Path Loss Exponent & 3.5 \\
Area of monitoring field (meters) & 500 meter x 500 meter \\
Performance Metrics that being measured & BER, Remaining Energy \\
& $(\mu \mathrm{W})$ and Latency (ms) \\
\hline
\end{tabular}

\subsection{Measurement Models}

In this paper, we demonstrated several formulas that had been used for the measurements throughout the experiments. The expression in Equation (1) shows the formula of the error correcting capability, t of RS code:

$$
2 \mathrm{t}=\mathrm{n}-\mathrm{k}
$$

where $\mathrm{n}$ is the codeword length and $\mathrm{k}$ is the number of data symbol [8]. We also formulate the BER for BPSK [9] as in Equation (2) which includes Rayleigh Fadings:

$$
\mathrm{BER}=\frac{1}{2}\left(1-\sqrt{\frac{\frac{E_{b}}{N_{0}}}{\frac{E_{b}}{N_{0}}+1}}\right)
$$

While, the received signal power expression in signal processing denoted in Equation (3):

$$
Y=h(x)+n
$$

wherehdenoted the channel gain, $x$ is transmitted data and nis noise. 
Equation (4) shows the expression of received signal power, $\mathrm{Y}$ in Additive White Gaussian Noise (AWGN) that which cooperated the free space path loss principle. Here, we have added the path loss exponent and the distance between transmitter and receiver [10]. Where $\alpha$, the path loss exponent such that $2 \leq \alpha \leq 6$ while $d$ is defined as the distance between transmitter and receiver (in meters).

$$
\mathrm{Y}=\mathrm{h}\left(\mathrm{d}^{\frac{-\alpha}{2}}\right)
$$

Equation (5) shows the expression of minimum energy, $\mathrm{E}_{\min }$. One transmitted bit consume one unit of energy while one received bit would consume 0.75 units of energy:

$$
E_{\text {min }}=H \times N_{p} x\left(N_{b i t s}+N_{\text {bits }} \times 0.75\right)(5)
$$

where $\mathrm{H}$ is the number of hops, $N_{p}$ is the number of packets, and $N_{b i t s}$ is the total number of bits. We assumed that the number of hops is one for every experiment in this paper as for the single-hopping communication. Thus, the equation for energy consumed in a transmission that implements CRC-4 and Error correcting codes, $\mathrm{E}_{\mathrm{Ecc}}$ can be rewritten as follows in Equation (6):

$$
E_{E c c}=H \times N_{p} x\left(N_{b i t s}+\left(N_{b i t s} \times 0.75\right)+E_{D e c}(6)\right.
$$

Based on Equation (6), the decoding energy, $\mathrm{E}_{\mathrm{Dec}}$ can be calculated as follows:

$$
E_{\text {Dec }}=\left(2 n t+2 t^{2}\right)\left(E_{\text {addition }}+E_{\text {multiplication }}\right)
$$

where $\mathrm{n}$ is block length for error correcting codes and $\mathrm{t}$ is error correcting capability. While, $E_{\text {addition }}+E_{\text {multiplication }}$ is the energy consumed in the process of addition and multiplication of error correcting codes. We do not include encoding energy as encoding energy can be negligible small [11].

Based on Equation (8), the network latency can be expressed as:

$$
\text { Latency }=\text { Delay }_{\text {Prop }}+\text { Delay }_{\text {Trans }}
$$

where Delay ${ }_{\text {Prop }}$ is the propagation delay and Delay ${ }_{\text {Trans }}$ is the transmission delay or also known as serialization delay [12-13]. The Propagation delay formula also expressed in equation (9):

$$
\text { DelayProp }_{\text {Pro }} / \mathrm{s}(9)
$$

where $\mathrm{d}$ is the distance between sender and receiver and $\mathrm{s}$ is propagation speed of the media. While, the Transmission delay is calculated as below in Equation (10):

$$
\text { Delay }_{\text {Trans }}=\mathrm{L} / \mathrm{R}
$$


where $\mathrm{L}$ is the length of a packet in bits while $\mathrm{R}$ is the transmission rate.

\section{RESULTS AND DISCUSSION}

The results of the implementation of MEC algorithm wereanalyzed and discussed to study the effects of different error correction schemeto that correspond with MEC on the number of nodes and bit length towards BER, remaining energy and latency. The results obtained can be the references to further enhancing and for the optimizing the MEC algorithm to suit the configuration and architecture of CDMA WSN in terms of its functionality in error correcting capability, $\mathrm{t}$ of different codeword length, $\mathrm{n}$.

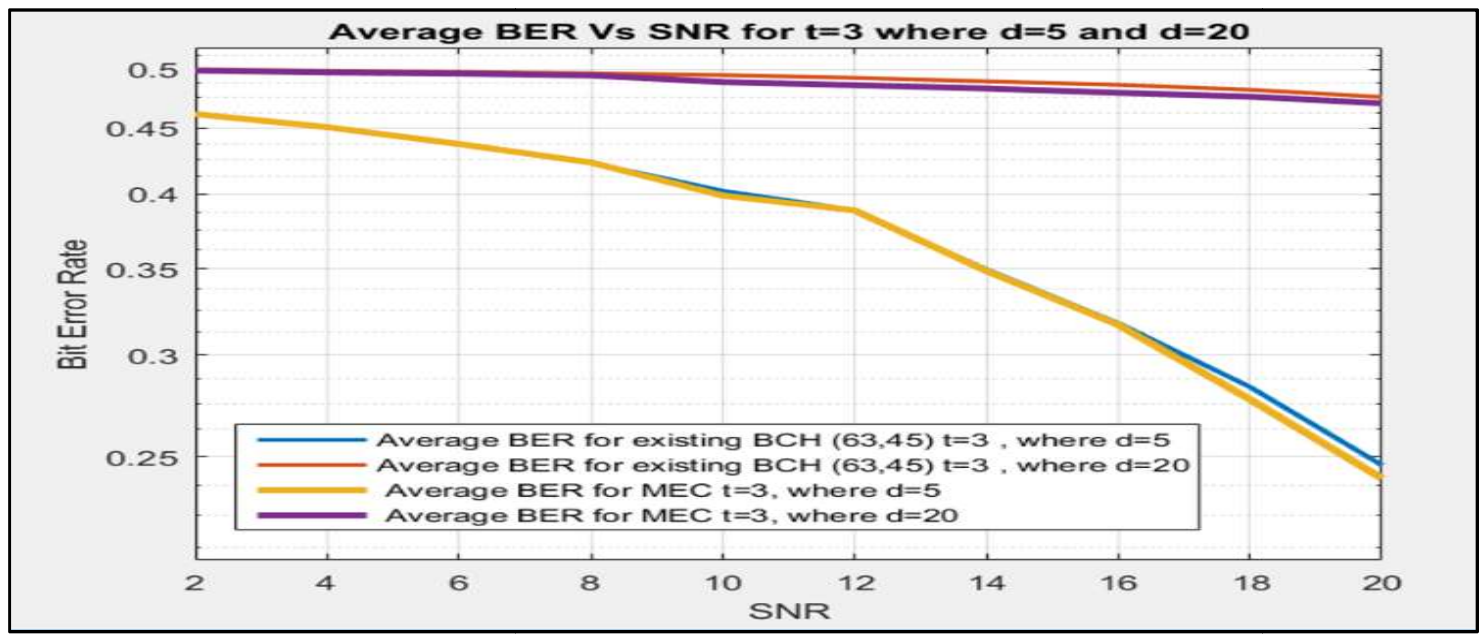

Fig.4. Graph of average BER vs. SNR for $d=5, d=20(t=3)$

Fig. 4 shows the graph of the comparison of average BER against SNR for same error correcting capability (Scenario 1 and Scenario 4) for $\mathrm{d}=5$ meters and $\mathrm{d}=20$ meters between existing $\mathrm{BCH}$ and MEC. From the graph, we know that as the distance between the sender and receiver increase, the BER will also slightly increases [14]. For $d=5$, we can see that MEC had outperformed existing $\mathrm{BCH}$ around SNR $=10$ and also MEC outperform existing $\mathrm{BCH}$ when SNR at its highest. Not to mention, MEC also outperforms existing BCH when $\mathrm{d}=20$ when SNR reaching to 8 until 20. This is because, from the testing, as the high number of correcting capabilities used, more bit of redundancy were appended to the data. For unconducive network environments where the highest number of nodes being deployed or high level of interference present, the existing error correction schemes might promote to increase in BER. While, MEC uses multiple error correction in which $\mathrm{BCH}$ or RS with 
different error correcting capability, $t$ will be implemented according to the change in network condition to actually optimize the number of bits that transmitted through the channel to reduce overhead.

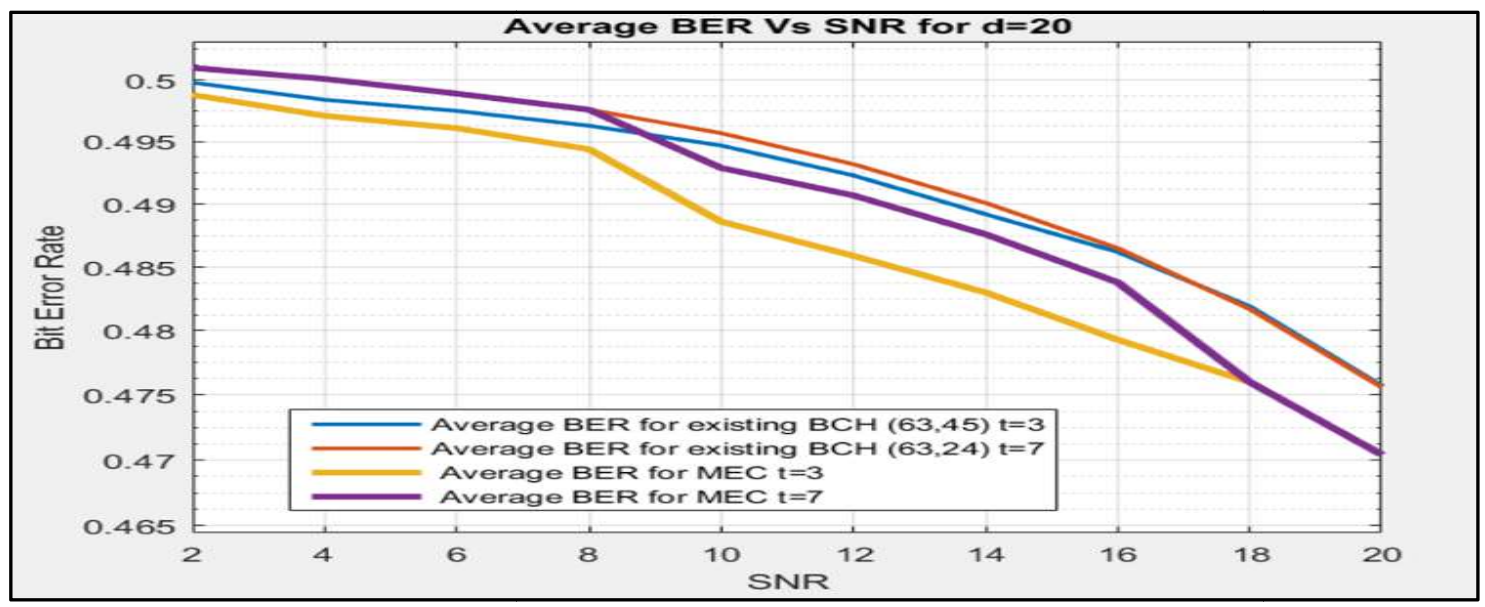

Fig.5. Graph of average BER vs. $S N R$ for $d=20(t=3$ and $t=7)$

Fig. 5 shows the graph of average BER against SNR for $d=20$. We test the existing $B C H$ of $(63,45)$ where $t=3$ and $\mathrm{BCH}$ of $(63,24)$ where $\mathrm{t}=7$ with MEC $\mathrm{t}=3$ and $\mathrm{t}=7$ (Scenario 4 and Scenario 6). Based on the graph, the existing $\mathrm{BCH}$ of $\mathrm{t}=3$ might outperform MEC $\mathrm{t}=7$ when $\mathrm{SNR}$ is at 2 until 9. However, the existing $\mathrm{BCH}$ of $\mathrm{t}=3$ and $\mathrm{t}=7$ have higher BER even when SNR is at its highest such when SNR at 10 until 18. The MEC when $\mathrm{t}=3$ have the lowest BER compared to other schemes. This is because, the lower number of redundancy append to the data lower the congestion or flooding of bits on the network.

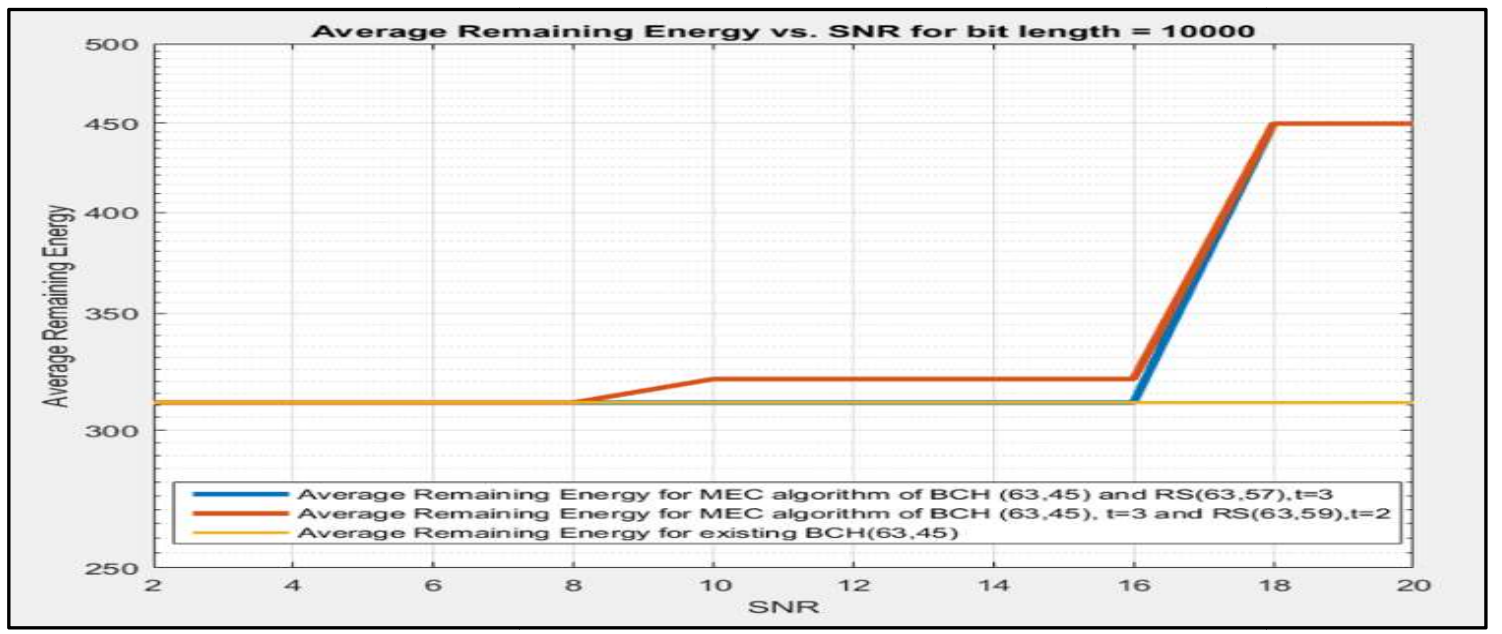

Fig.6. Graph of average remaining energy vs. SNR for bit length $=10000(t=3$ and $t=2)$ 
Fig. 6 illustrated the graph of average remaining energy against increasing SNR when bit length is 10000 . We tested the existing $\mathrm{BCH}(63,45) \mathrm{t}=3$ with $\mathrm{MEC} \mathrm{BCH}$ RS $\mathrm{t}=3$ and MEC $\mathrm{BCH} t=3 \mathrm{RS} t=4$. The MEC $\mathrm{BCH}$ RS $\mathrm{t}=3$ shows the same value of remaining energy as existing $\mathrm{BCH} t=3$ when SNR at 2 until 16 . When the SNR reaching 16 to 20 , the remaining energy increases as the retransmissions will take place instead of error correcting codes. The graph concluded that existing $\mathrm{BCH}$ has lower remaining energy regardless of SNR. We cooperated the use of retransmission at the higher SNR (where likely less network congestion) to lower the overhead of decoding and redundancy added to the network. Not to mention, when SNR is at its highest, where we assumed network condition is in good condition, retransmission is likely more sufficient to correct corrupted bits with error correcting codes.

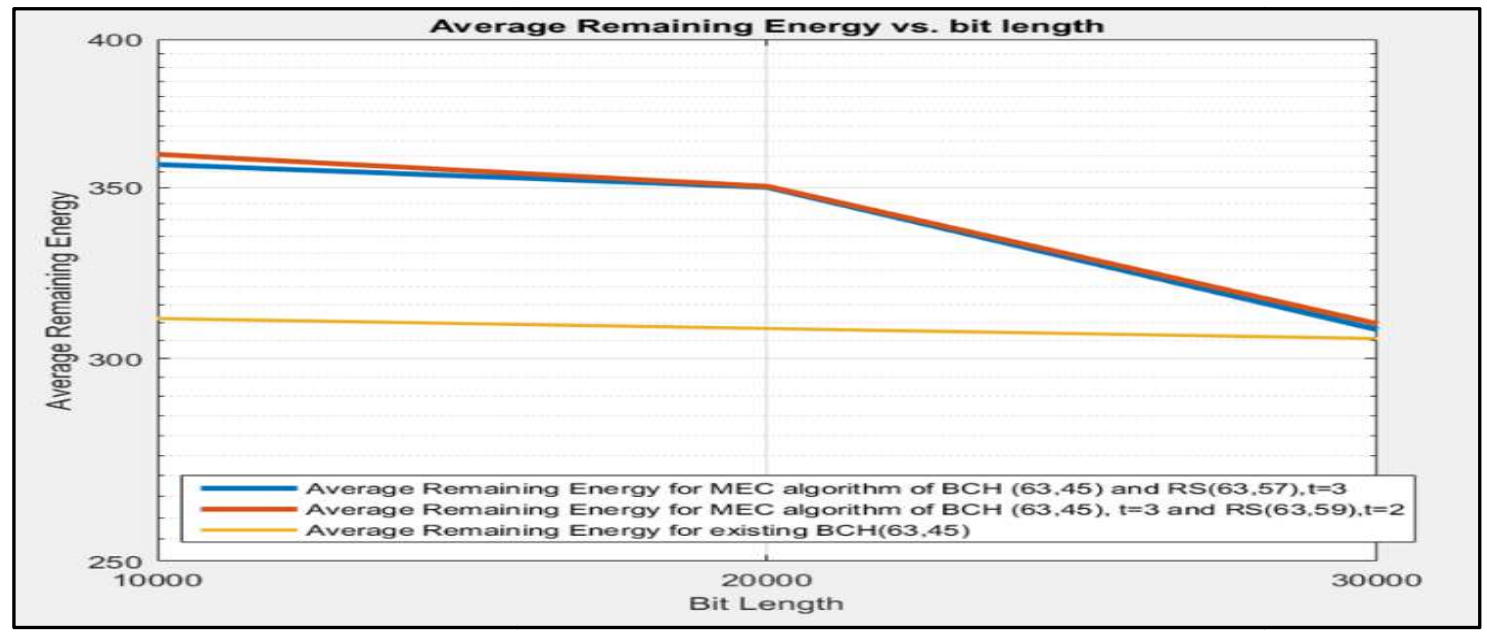

Fig.7. Graph of average remaining energy vs. bit length $(t=3$ and $t=2)$

Fig. 7 shows the graph of average remaining energy against increasing bit length of 10000 , 20000 and 30000 bits. As the bit length increases, the more energy needed to be used to transmit a single packet. From the graph, the existing $\mathrm{BCH}$ has lower remaining energy compared to MEC when bit length increases from 10000 to 30000 such that $311.0888 \mu \mathrm{W}$, 308.2987 $\mu \mathrm{W} 305.5233 \mu \mathrm{W}$ respectively. While, MEC BCH t=3 RS $\mathrm{t}=2$ have the highest remaining energy of $360.6350 \mu \mathrm{W}$ at low bit length as well as MEC BCH RS $t=3$ where remaining energy is at $357.3480 \mu \mathrm{W}$. The performance of BER when MEC BCH t=3 RS t=2 might be slightly lower than existing $\mathrm{BCH}$, but still can optimize between the BER and remaining energy compared to existing $\mathrm{BCH} \mathrm{t}=3$.

Fig. 8 shows the graph of latency against increasing number of nodes around 12 nodes to 25 
nodes approximately. We tested the number of nodes from 4 nodes to 64 nodes and found that the latency of the network when $\mathrm{d}=5$ and $\mathrm{d}=20$ were much higher when existing $\mathrm{BCH}$ was implemented as compared to MEC $\mathrm{t}=3$ when $\mathrm{d}=5$ and $\mathrm{d}=20$. MEC $\mathrm{t}=3$ have the lowest overall latency when the number of nodes increases. The MEC had outperformed the performance of reducing overall latency as the number of nodes increases compared to existing $\mathrm{BCH} t=3$. This is because the MEC uses RS $(63,57)$ when SNR from 9 to 16 . RS codes in MEC have lower latency than $\mathrm{BCH}$ codes due to its lower capability to correct errors than $\mathrm{BCH}$. However, from the graph of BER illustrated in Fig. 4 and Fig. 5, at the medium network condition during SNR from 9 to 16, it was shown that the RS can also correct errors as well as $\mathrm{BCH}$ as the BER had been optimized and reduced significantly.

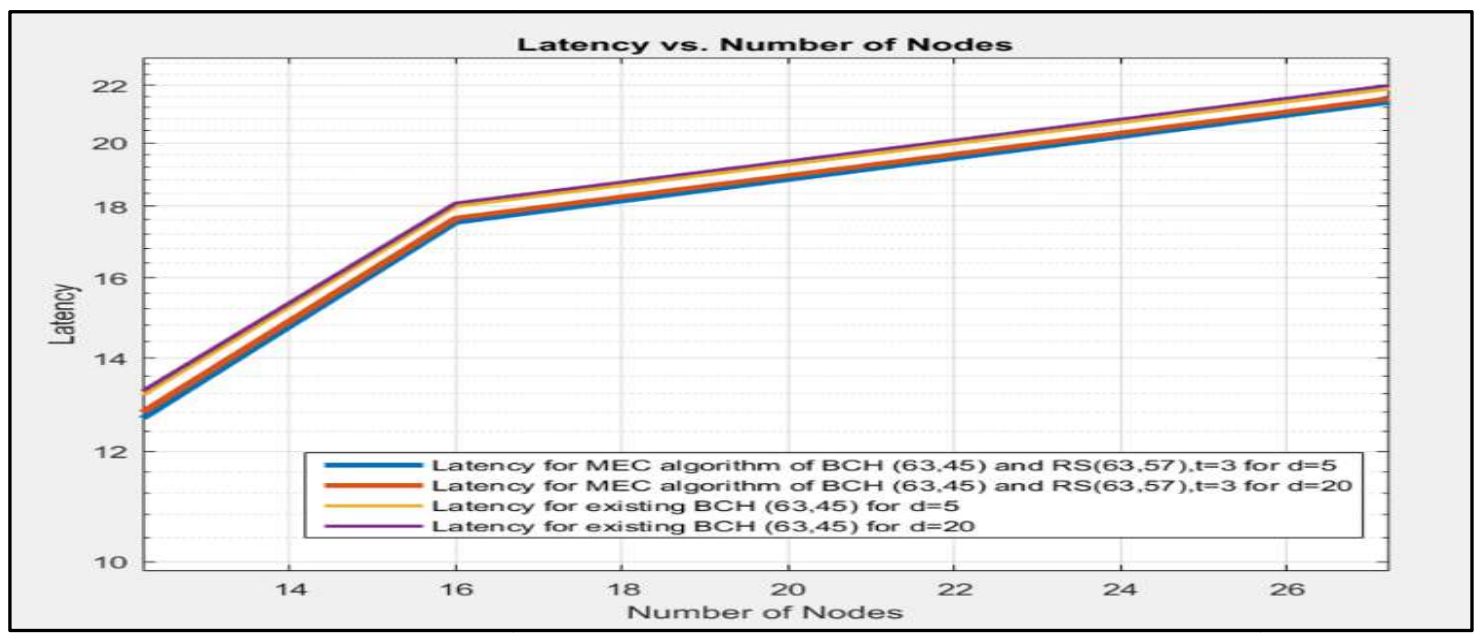

Fig.8. Graph of latency vs. number of nodes for $\mathrm{t}=3(\mathrm{~d}=5$ and $\mathrm{d}=20)$

\section{CONCLUSION}

From the results obtained in the previous section, we can conclude that MEC can outperform existing error correction schemes as MEC consists of multiple error correction that corresponds to the changing of network conditions. It is important to cooperate the network condition as it is always changing over time. The use of inaccurate error correcting codes with such high error correcting capabilities on the congested network might lead to much higher BER and higher energy consumption. The idea of extending the HARQ process to allow such multiple error correction to be used to boost the lifetime of the wireless sensor network for crucial activities such as natural disaster monitoring where the lifetime need to be at the 
maximum to keep the network alive. BER and latency also need to be kept as low as possible to promote accurate data to be transmitted to the sink so that the disaster detection and early warning can be given to the public at the accurate time.

This research is the work in progress that follows the work of $[6,15]$. This paper only cooperated the used of block codes such as $\mathrm{BCH}$ and RS and retransmission strategy. For future work, MEC hopefully can be tested with convolutional codes as well as other common ECC such as Hamming or LDPC to test this ECC performance with MEC for predefined topology.

\section{ACKNOWLEDGEMENTS}

This research is supported by the Research Management Institute, UniversitiTeknologi MARA and registered under the Fundamental Research Grant Scheme (FRGS) \#600-RMI/FRGS 5/3 (46/2015).

\section{REFERENCES}

[1] Deshmukh P V M, Pathan S C, Chanvan S V, Tilekar S K, Ladgaonkar B P. Wireless sensor network for monitoring of air pollution near industrial sector. International Journal of Advanced Research in Computer Science and Software Engineering, 2016, 6(6):638-645

[2] SudarsonoA, Huda S, Fahmi N, Al-Rasyid M U H, Kristalina P. Secure data exchange in environmental health monitoring system through wireless sensor network. International Journal of Engineering and Technology Innovation, 2016, 6(2):103-122

[3] Xu G, Shen W, Wang X. Applications of wireless sensor networks in marine environment monitoring: A survey. Sensors, 2014, 14(9):16932-16954

[4] Datta U, Sen S, Kundu S. Energy level performance of HARQ-II scheme in CDMA wireless sensor network with correlated interferers. In Annual IEEE India Conference, 2011, pp. $1-4$

[5] Datta U, Kundu S. Performance of multi-hop CDMA wireless sensor networks with correlated interferers using different retransmission strategies and error control schemes. International Journal of Sensor Networks, 2014, 15(1):40-51

[6] Razali S, Mamat K, Bashah N K. A new approach for wireless sensor network lifetime 
maximization and low overhead with hybrid ARQ (HARQ) error control protocol. Australian Journal of Basic and Applied Sciences, 2015, 9(26):36-41

[7] Manzoor B, Javaid N, Rehman O, Bouk S H, Ahmed S H, Park S H, Kim D. Energy aware error control in cooperative communication in wireless sensor networks. ACM SIGAPP Applied Computing Review, 2014, 14(3):55-64

[8] ShrivastavaP, Singh U P. Error detection and correction using Reed Solomon codes. International Journal of Advanced Research in Computer Science and Software Engineering, $2013,3(8): 319-323$

[9] Meghdadi V.

BER calculation.

2008 ,

http://www.unilim.fr/pages_perso/vahid/notes/ber_awgn.pdf

[10] Weber S, Andrews J G, Jindal N. The effect of fading, channel inversion, and threshold scheduling on ad hoc networks. IEEE Transactions on Information Theory, 2007, 53(11):4127-4149

[11] Alrajeh N A, Marwat U, Shams B, Shah S SH. Error correcting codes in wireless sensor networks: An energy perspective. Applied Mathematics and Information Sciences, 2015, 818(2):809-818

[12] University of Minnesota. Network delays and losses. 2017, https://www.d.umn.edu/ gshute/net/delays-losses.xhtml

[13] RF Wireless World. Network latency calculator. 2010, http://www.rfwireless-world.com/calculators/Network-Latency-Calculator.html

[14] MatarnehAM. Thorough investigation of BER simulation of DPSK in underwater acoustic channel. Jordan Journal of Electrical Engineering, 2016, 2(2):160-171

[15] Razali S M, Mamat K, Abdul B K, Ali F H M. Performance enhancement of wireless sensor network (WSN) with the implementation of Hybrid ARQ (HARQ) and transmission power control (TPC). In IEEE Conference on Wireless Sensors, 2014, pp. 36-40

\section{How to cite this article:}

Razali S, Mamat K, Bashah S K. Performance of multiple error correction (mec) scheme based hybrid arq (harq) algorithm for maximizing lifetime of wireless sensor network (wsn) for natural disaster monitoring. J. Fundam. Appl. Sci., 2017, 9(5S), 336-351. 